\title{
Upending Racism in Psychological Science: Strategies to Change How Science is Conducted, Reported, Reviewed \& Disseminated
}

\author{
NiCole T. Buchanan ${ }^{1}$, Marisol Perez ${ }^{2}$, Mitchell J. Prinstein ${ }^{3}$, and Idia B. Thurston ${ }^{4}$ \\ ${ }^{1}$ Department of Psychology, Michigan State University \\ ${ }^{2}$ Department of Psychology, Arizona State University \\ ${ }^{3}$ Department of Psychology and Neuroscience, University of North Carolina at Chapel Hill \\ ${ }^{4}$ Department of Psychological and Brain Sciences; Department of Health Promotion and Community \\ Health Sciences, Texas A\&M University
}

This is a post-peer-review, pre-copyedit version of the following (please use the citation below): Buchanan, N. T., Perez, M., Prinstein, M., \& Thurston, I. (2021). Upending Racism in Psychological Science: Strategies to Change How Our Science is Conducted, Reported, Reviewed, and Disseminated. American Psychologist, 76(7), 1097-1112. https://doi.org/10.1037/amp0000905

\section{Author Note}

NiCole T. Buchanan (https://orcid.org/0000-0001-9288-5245), Marisol Perez (http://orcid.org/00000002-0915-2145), Mitchell J. Prinstein (https://orcid.org/0000-0002-7587-8665), and Idia B. Thurston (https://orcid.org/0000-0003-2484-2985).

We have no known conflicts of interest to disclose. Authorship is listed in alphabetical order by last name because, all authors contributed equally. Authors self-identify as a U.S. born biracial Black woman, Hispanic woman, white male, and Nigerian American Immigrant Black woman. Correspondence regarding this manuscript may be directed to Dr. Idia Thurston, Texas A\&M University, 256 Psychology Building, College Station, TX 77843, idiathurston@tamu.edu, 979-845-3794

\section{See related manuscript:}

Buchanan, N. T., Perez, M., Prinstein, M., \& Thurston, I. (2021). Diversity Accountability Index for Journals (DAI-J): Increasing awareness and establishing accountability across psychology journals [unpublished manuscript]. PsyArXiv. https://psyarxiv.com/zp9em 


\begin{abstract}
As efforts to end systemic racism gain momentum across various contexts, it is critical to consider antiracist steps needed to improve psychological science. Current scientific practices may serve to maintain white supremacy with significant and impactful consequences. Extant research practices reinforce norms of homogeneity within BIPOC (Black, Indigenous, and other People of Color) populations, segregate theories and methods derived from BIPOC groups, apply disparate standards to the evaluation of research on white vs. BIPOC populations, and discourage BIPOC scholars from pursuing research careers. Perhaps consequently, disparities persist on a range of psychologically relevant outcomes (e.g., mental and physical health). This article presents examples of how epistemic oppression exists within psychological science, including in how science is conducted, reported, reviewed, and disseminated. Importantly, this paper offers a needed contribution by providing specific concrete recommendations for different stakeholders, including those involved in the production, reporting, and gatekeeping of science as well as consumers of science. Additionally, a discussion of accountability steps are offered to ensure that psychological science moves beyond talk and towards action, with possible strategies to measure outcomes, stimulate progress, promote dialogue and action, challenge inequity, and upend the influence of white supremacy in psychological science.
\end{abstract}

Keywords: racism, psychological science, epistemic oppression, research dissemination, social justice

Public Health Statement: Challenging systemic racism requires multi-systemic change. In this paper, potential solutions and measurable outcome metrics to eliminate white supremacy in psychological science are provided. 
Upending Racism in Psychological Science: Strategies to Change How Science is Conducted, Reported, Reviewed \& Disseminated

Recent national attention to historical and contemporary racism, spurred by the killings of Breonna Taylor, George Floyd, and countless Black community members in the United States (Proctor, 2020) have spurred critical examination of the role of systemic racism in all sectors of society.

Psychology has a long history of promoting social justice initiatives (Leong et al., 2017), but has made insufficient progress in promoting socially just outcomes, particularly in rooting out racism within institutional practices (Miller et al., 2019b; Winston, 2020). Racism often is defined at the individual level as psychological processes that contribute to stereotyping, prejudice, and discrimination (Salter et al., 2018). However, focusing on individual-level factors can mask how cultural and institutional processes contribute to racism and maintain white supremacy ${ }^{1}$ (Salter et al., 2018; Roberts \& Rizzo, 2020; Syed \& McLean, 2021). For example, focusing on revising a single rejected manuscript on BIPOC communities can ignore findings that diversity-related manuscripts experience disparate review processes (King et al., 2018) and may be devalued broadly in psychology (Guthrie, 2004; Stevens et al., 2021). Changing systemic racism requires multi-systemic change. This paper identifies several systems and stakeholders requiring reform to eliminate white supremacy in psychological science, and importantly, offers concrete, measurable actions with suggested accountability steps to move from the identification of barriers to specific action steps for change.

This paper is guided by the belief that epistemic oppression (i.e., systematic exclusion that hinders contribution to knowledge production and advancement; Dotson, 2012) within psychological science limits and creates inherent flaws in research (Buchanan \& Wiklund, 2020, 2021; Settles, Warner et al., 2020). Thus, efforts to eliminate epistemic oppression are necessary to facilitate a psychological science that is unbiased, representative, and maximally impactful. To date, psychological research struggles to incorporate or demonstrate adequate generality to BIPOC populations (Burlew et al., 2019;

\footnotetext{
${ }^{1}$ We have intentionally chosen to capitalize Black to indicate the shared culture and history of Black and African American people in the United States and to raise awareness about the marginalization of Black identity. We use lower case to refer to "white" and "white supremacy" because capitalization of the word "white" may in itself be an example of white supremacy (see https:/www.cjr.org/analysis/capital-b-black-styleguide.php for more information).
} 
Hall et al., 2016), and has relied predominantly on theoretical models developed by white scholars for white populations (Buchanan and Wiklund, 2021; Guthrie, 2004; Markus, 2008; Settles, Warner, et al., 2020; Syed et al., 2018; Zuberi \& Bonilla-Silva, 2008). For example, sexual harassment research has mainly focused on the experiences of white women with less attention to how race and gender intersect to create unique experiences for women of color with differential effects on their well-being (e.g., racialized sexual harassment; Buchanan \& Ormerod, 2002; Buchanan et al., 2018). As a consequence, limited progress has been made to substantially reduce physical or mental health disparities across racial and ethnic groups (see Baker et al., 2010; Buchanan \& Wiklund, 2020; Burlew et al., 2019). Moreover, BIPOC populations remain significantly underrepresented among journal editors, associate editors, and reviewers (over 90\% of which are white; Dawson et al., 2020; Greco et al., 2016; Taylor et al., 2020), and psychological studies that address race by white researchers tend to use primarily white samples (and smaller proportions of BIPOC participants) compared to samples of BIPOC scholars (Roberts et al., 2020). Furthermore, in some journals research conducted by BIPOC scholars, or on predominantly BIPOC samples, are twelve times more likely to be rejected than accepted (King et al., 2018) and when published, these articles are frequently segregated in "specialty journals" while research by white scholars focused on predominantly white populations has a greater chance of being published in higher impact outlets (Hall \& Maramba, 2001; Hartmann et al., 2013; Roberts et al., 2020). These and many other indicators suggest that psychological science reflects a systemic pattern of white supremacy.

Change in psychological science requires a responsibility and commitment to antiracist action, actions toward reducing racism, and "proactive antiracism" where racism is challenged before it occurs (Roberts \& Rizzo, 2020, p. 10). Toward that end, this paper provides specific recommendations (for a summary see Supplemental Table 1) to dismantle white supremacy in how psychological science is conducted, reported, reviewed and disseminated that are applicable to authors, educators, journal editors/publishers, and reviewers. This paper focuses on changes in the publication processes as "racism is pervasive in scholarly publishing," and entrenches biases throughout the discipline (Dawson et al., 2020, p. 170). 


\section{How Science is Conducted}

Research focusing on BIPOC populations is disproportionally less than research on white populations (Hall \& Maramba, 2001; Hartmann et al., 2013; Roberts et al., 2020; Syed et al., 2018), and studies that focus on BIPOC communities often require a white comparison group to be published, particularly in higher impact journals (see Stanley, 2007, and Wang, 2016 for examples). Notably, this same standard (i.e., requiring BIPOC comparison groups) is often not applied to studies of predominantly white populations (Cundiff, 2012; Hall \& Maramba, 2001; Hartmann et al., 2013, Roberts et al., 2020). Current research tends to utilize recruitment and hypothesis-generation approaches that reflect predominantly white convenience samples (e.g., from introductory psychology classes at predominantly white institutions in the United States; Arnett, 2008; Guthrie, 2004; Henrich et al., 2010; Thalmayer et al., 2020). These approaches can impact how research is conducted and analyzed, permitting substantive examination of white within-group differences while precluding the examination of BIPOC within-group differences. Furthermore, such practices prioritize conducting research with, by, and for white people, which reinforces whiteness as a normative default and can communicate that BIPOC people are atypical, less important to study, and less valuable relative to others (Held, 2020; Teo, 2008; Winston, 2020). Accordingly, upending racism in psychological science will require several changes to the ways in which research is conducted.

1. Conduct and publish more research on race. The paucity of research on BIPOC communities, particularly in "mainstream" (i.e., higher impact) journals, is thought to directly affect citation metrics of scholars who study these topics, the impact of relevant papers, and even the science pipeline of BIPOC scholars. Thus, the lack of papers on BIPOC communities becomes a self-perpetuating issue in the field. Over $90 \%$ of publishing editors are white (Greco et al., 2016; Taylor et al., 2020) and given that $84 \%$ of psychologists identify as white (APA, 2018), the pool of potential reviewers is also overwhelmingly white. Scholars who focus on BIPOC populations and BIPOC-related topics thus are underrepresented in the manuscript review processes, particularly within "mainstream" (i.e., high impact) publications, meaning that submissions to these higher impact outlets continue to be evaluated predominantly by white scholars and editors (Greco et al., 2016). Perhaps consequently, BIPOC scholars are more likely to 
publish papers in "specialty" outlets, and less likely to be cited by white scholars (Bertolero et al., 2020; Chakravartty, et al., 2018), which can have negative implications for hiring and promotion, particularly at universities that value citation indices. In turn, fewer BIPOC scholars are available to recruit graduate students who will pursue research careers, thus maintaining a major gap in the field.

These are high priority issues for change (Roberts et al., 2020). The exclusion of BIPOC scholarship and the interpretation of findings in ways that harm BIPOC communities are non-neutral acts of epistemic violence and oppression (Dotson, 2012; Teo, 2008) with potentially harmful consequences not only to BIPOC communities, but also to the future of psychological science and its societal impact (Buchanan \& Wiklund, 2020; Osbeck, 2018). It is recommended that journals seek out publications on BIPOC populations by encouraging special issues that raise awareness about these concerns (e.g., see Valrie et al., 2020). Journals should also showcase studies and call for papers that center all of the experiences of BIPOC communities. This means publishing balanced stories about the full experiences of Black and Brown people, not only publishing negative outcomes, and across papers, ensuring that a full spectrum of the population is represented (e.g., not only low-income members of a group). Journals should champion publications on BIPOC samples, support scholarship on topics relevant to BIPOC communities, and report improvements over time for accountability (i.e., suggested benchmarks are provided in a draft diversity accountability index (Buchanan et al., 2021) at https://psyarxiv.com/zp9em). After establishing baseline data on the representation of these topics and samples, it is recommended that each journal establish benchmarks to ensure yearly increases in the number of publications on these critical topics.

There also is an urgent need for more equitable funding processes, particularly for federal granting agencies, such as the National Institutes of Health (NIH) and National Science Foundation (NSF) (Ginther et al., 2011; Hoppe et al., 2019; Stevens et al., 2021). Grant selection processes continue to reveal biases that can harm BIPOC scholars and the study of BIPOC populations, and the lack of BIPOC scholars on grant review panels continues to be a significant concern. Significant disparities in NIH funding persist, with Black scholars receiving funding at approximately half the rate of white scholars (Dzirasa, 2020; Erosheva et al., 2020; Ginther et al., 2011) and over 20\% of the NIH funding disparities 
for Black scholars being related to their topic foci, such as health disparities, even after controlling for other relevant variables (Hope et al., 2019). This can have negative implications for promotion in academia, as many universities value obtaining a NIH grant as part of the promotion process. Special program announcements to examine underrepresented populations offer an excellent opportunity to increase research on these topics. Federal, state, local, and private funding institutes could dedicate funds for research on BIPOC populations, train grant reviewers regarding diversity science and related methodologies, and introduce new grant mechanisms that recognize the unique challenges inherent in community-based research and other approaches that successfully recruit and retain BIPOC study participants. Public accountability when harm to BIPOC populations is caused by psychological research would also be an important initial step to rebuilding trust and healing the damage caused to BIPOC communities.

2. Exemplify values regarding diversity, equity, and inclusion. The production of science is substantially influenced by journal and funding agency priorities. Thus, journals and editorial staff must move beyond diversity statements that express solidarity with BIPOC scholars or simply state 'Black Lives Matter' and instead demonstrate that they value scholarship that benefits BIPOC communities and scholars. Toward this goal, publishers could invest in training and educating their editorial staff to recognize the added value of publishing BIPOC-focused manuscripts and highlight this expectation when inviting editorial board members and reviewers to serve. Journal mission statements should include language that reflects values related to diversity, equity, and inclusion, in science, highlight a desire for more research on within-group differences among BIPOC populations in journals that publish empirical papers (for example, see Cultural Diversity and Ethnic Minority Psychology Journal), showcase these papers when they are published (e.g., see Springer Nature https://www.springernature.com/gp/researchers/ campaigns/black-lives-matter for an example), and promote the visibility of BIPOC scholarship (e.g., website spotlights or twitter posts from the journal's official handle). This could be done by requiring author positionality statements regarding social identity, such as the acknowledgment section of this paper. This could serve as informational representations of diversity and inclusion. 
BIPOC scholars are also underrepresented in scholarly citations as scholars determine which papers and journals to include in reference lists (Chakravartty et al., 2018; Bertolero et al., 2020). Citation rates are positively associated with journal impact factors (Lozano et al, 2012), presenting a challenge for BIPOC scholarship when it is less likely to be included in high impact journals. An examination of 25 years of papers across five journals revealed that citation practices of white authors (e.g., a strong preference for citing only white authors and self-citations) not only can drive these racial inequities in citations, but continue even with increased diversity in the field (Bertolero et al., 2020). Moreover, Bertolero and colleagues (2020) documented that BIPOC women were significantly less likely to be included in reference lists compared to white or BIPOC men and white women. There are ways to address these disparities. For example, Zurn and colleagues (2020) propose a citation diversity statement be added to papers that discuss authors' attempts to cite equitably and the resulting (gender) diversity of the authors referenced. If this was expanded to include BIPOC scholars, it may increase the diversity of scholars cited to the extent author social identities are known. Additionally, paper page limits can impact author behavior (Card \& DellaVigna, 2012), and perhaps increase the tendency to eliminate diverse scholarship. Given tendencies to cite ingroup members and exclude outgroup members' scholarship (Greenwald \& Schuh, 1994; Chakravartty et al., 2018; Bertolero et al., 2020), relaxing page limit requirements for reference lists could reduce the likelihood that authors will achieve page limitations by omitting BIPOC scholarship.

3. Use research methods most appropriate for BIPOC populations. Methods for recruiting and examining questions among white populations have been used almost exclusively within psychology for over a century (Guthrie, 2004). Researchers should routinely evaluate the appropriateness of methods and questions used in research with BIPOC individuals. For example, only recently has the Boston Naming Test, a neuropsychological assessment, removed the noose item due to its offensive nature (Attridge et al., 2020). For some research, community-based participatory research (CBPR) strategies (Israel et al., 2010) can offer a complementary approach with research theories and methods (i.e., measures, paradigms) that better reflect BIPOC individuals and their lived experiences, address health disparities, and add novel contributions to the research literature. However, CBPR is not immune to 
racism, and Chavez and colleagues (2008) provide recommendations to implement CBPR in ways that empower community partners of color as equal partners in community research settings. Further, person-centered quantitative approaches (as compared to variable-centered) analyses (Howard \& Hoffman, 2018) may better capture heterogeneity within diverse populations and the intentional use of mixed-method approaches to scientific inquiry can elevate BIPOC voices, document multiple perspectives in research endeavors, and expand strength-based inquiry for BIPOC communities (Schneider, 1998). To achieve these goals, graduate student research training should include approaches that encourage greater representation of the diversity of BIPOC communities and journals should communicate that they value diverse methods of inquiry that are more suitable for diverse populations. 4. Encourage recruitment of more diverse samples. Papers using predominantly white samples are typically published without identifying their sample in the title, providing a rationale for including only white participants, or framing the study as having limited generalizability due to the homogenous sample (Guthrie, 2004; Henrich et al., 2010; Thalmayer et al., 2020). In contrast, studies with predominantly BIPOC samples are often required to indicate so in the title, justify their sample, and discuss the limitations of the study's generalizability to the broader society. Reviewers are also more likely to require that papers with BIPOC samples include a white comparison group to be published (Stanley, 2007; Wang, 2006). These practices can both perpetuate and reify the belief that whiteness is the norm by which all others must be compared (Cundiff, 2012; Stanley 2007) and they create undue burdens on BIPOC-focused research and scholars. Moreover, such practices effect how science is conducted, and can permit researchers with white samples to ignore the need to diversify their samples, and can compel those working with BIPOC samples to recruit a white comparison group, even when there is no scientifically-driven reason to do so (Cundiff, 2012; Hall \& Maramba, 2001; Hartmann et al., 2013; Markus, 2008; Stanley, 2007).

Psychological science is advanced when investigators consider innovative solutions to diversifying their samples and research teams. This includes work that balances power and amplifies the strengths of the team, such as academic collaborations between predominantly white institutions (PWIs), where research resources tend to be higher, and Historically Black Colleges and Universities 
(HBCUs), Hispanic Serving Institutions (HSIs), and Tribal Colleges and Universities where diverse college populations tend to be higher. Similarly, collaborations with community colleges, which tend to have more diverse student populations, can lead to a more diverse human subject pool and community partnerships with BIPOC organizations can increase trust and participation in research studies. Journals can facilitate changes in how investigators collect data by establishing minimum requirements for sample diversity in papers that claim to represent the general population and require authors to justify their use of WEIRD samples (Western, Educated, Industrialized, Rich, and Democratic; Henrich et al., 2010) versus BIPOC samples and discuss generalizability limitations due to their sample. Further, manuscripts with WEIRD samples should indicate so within paper titles and reviewers should assess manuscripts for concerns regarding inclusion, similar to federal grant reviews, to prompt investigators to be cognizant of addressing these issues when conducting research. Such requirements begin to address systemic racism, by shifting away from centering whiteness as a universal norm and better identifying the generalizability of each manuscript. Over time, these recommendations could increase the visibility of BIPOC studies, increase BIPOC-centered submissions, utilization of BIPOC reviewers' expertise, and reduce discriminatory statements across reviews.

5. Promote Diversity Science research approaches. Journals should encourage research that integrates a Diversity Science approach. Diversity Science is a field with a rich history and expansive application to psychological science (Miller et al., 2019a). Defined as "the study of the interpretation and construction of human difference - of why and how difference makes a difference--within the context of existing, historically shaped cultural and structural realities" (Plaut, 2010b, p. 168), Diversity Science refers to a set of practices for conceptualizing, conducting and reporting science. A Diversity Science approach moves away from prioritizing generality and focuses instead on identifying where differences exist, the sociocultural forces that lead to and reinforce those differences (Plaut, 2010a), and responsible practices to examine group differences appropriately (see Burlew et al., 2019; Matsumoto et al., 2001; Wang, 2016). Diversity Science research on race and racism can expand theory-informed research and evidence-based practice (Miller et al., 2019a; Hall et al., 2016). More broadly, Diversity Science provides methodological frameworks for improving psychological science and ensuring that it addresses the 
needs of a diverse global population. As Diversity Science has increased in popularity, it has also been misinterpreted to assume that the study of any and all differences "count." This reflects a form of theory-flattening (Alexander-Floyd, 2012) that is common as diversity- and social justice-related concepts move into the mainstream. This misuse of Diversity Science moves away from Plaut's (2010) definition, which emphasized the importance of a sociocultural understanding of racism and racial inequality, and reduces the potential impact of Diversity Science. Thus, psychology can monitor the use of the Diversity Science framework and resist the tendency to dilute it and render it more palatable and less impactful. To achieve these aims, scholars can learn about and incorporate Diversity Science theories and approaches, such as intersectionality theory, minority stress theory, and critical race studies, that center research within understandings of oppression and inequality (Buchanan \& Wiklund, 2021; Plaut, 2010a; 2010b; Miller et al., 2019a; Syed \& McLean, 2021; Zuberi, T., \& Bonilla-Silva, 2008). As a consequence, the introduction and discussion sections of manuscripts should illustrate how research questions and findings identify and illuminate systemic oppression, consider patterns of historical oppression and inequality, acknowledge the ways in which scientific knowledge has historically been used to benefit the privileged; and center study implications from an inequality and social justice lens (Buchanan \& Wiklund, 2020, 2021; Miller et al., 2019b; Rosenthal, 2016). Journals that allow diversity science manuscripts should clarify this in their submission options and consider adding language regarding Diversity Science to their mission statements to inform authors of their inclusivity.

\section{How Science is Reported}

The language academicians, writers, clinicians, editors, and publishers use matters. Words contribute to the maintenance of white supremacy by uplifting some while denigrating others. Labels can intentionally or unintentionally convey that one group is inherently inferior, or attribute blame to one's race rather than systems of inequity that have been inflicted upon (or power that has been taken away from) a racial group. The language used in psychological science often perpetuates negative stereotypes of BIPOC people, and can inadequately describe BIPOC samples and the contexts in which BIPOC identities are assessed. Upending racism in psychological science requires changes in how researchers report findings and the use of language that is more diverse, equitable, and inclusive. The 
Journal Article Reporting Standards published by APA establish the mandated reporting of race and ethnicity within a paper's Methods section (Applebaum et al., 2018; Levitt et al., 2018), which is important; however, to advance the reporting of psychological science four additional recommendations are offered below.

6. Require the use of system centered language. Today, many BIPOC people in the US live in lower income neighborhoods because operational structures and systems have disadvantaged these individuals. Yet, research often discusses risk factors attributed to a racial and ethnic group itself, rather than the conditions that members of this group have disproportionately experienced, or been unfairly disadvantaged by. Systems centered language (O'Reilly, 2020) includes a discussion of the current policies and historical roots that maintain health inequities, and the use of this language can promote a process of actively challenging narratives that have shaped how society views and treats BIPOC individuals. System centered language offers a paradigm shift for how language is used to describe BIPOC and other minoritized communities and is akin to person first language, where the person is put before the diagnosis (e.g., diabetic person vs. person with diabetes) to humanize the individual and show that the disease is but one aspect of their life. A similar model is proposed in systems centered language, such that the intergenerational systems that drive oppression and racism are held accountable and labelled appropriately so that the humanity of the individuals who are impacted by these systems is upheld. Using systems centered language encourages readers and authors to maintain conscious awareness that disparities are due to inadequate structures and processes rather than individual weaknesses. For example, instead of writing "BIPOC communities are vulnerable populations in the United States", write "BIPOC are among the most prohibited populations in the United States." Instead of writing "People living in poverty are at risk for poor health outcomes", write "People living in poverty are exposed to additional harms that drive poorer health outcomes" (see O'Reilly, 2020 for details). Similarly, when outcomes are presented (e.g., health disparities) and existing research is reviewed, findings should be framed within the systemic structures that maintain disparate outcomes. For example, when discussing negative health outcomes among Black populations, explain that these outcomes are due to systemic racism and discrimination, rather than stopping at the outcome. The 
current APA publication manual (APA, 2020), requires the use of person-first language and would advance the field with the addition of systems centered language requirements. Until then, journals can update author guidelines to require the use of systems centered language and have authors attest to the use of systems centered language in all submissions, and publicly report adherence (see suggestions in the draft diversity accountability index (Buchanan et al., 2021) at https://psyarxiv.com/zp9em).

\section{Update journal keywords to be more inclusive. Manuscript submission portals communicate} messages about the types of research that are valued. Many journals have established lists of keywords (Garcia et al., 2019) that fail to include categories related to diversity, equity, and inclusion. ${ }^{2}$ These omissions can communicate the (lack of) value the journal places on these topics. Further, research indicates that accurate and comprehensive lists of keywords that accurately reflect article topics are more effective in dominant search engines (such as Google Scholar), and increase article citations (Garcia et al., 2019). Thus, authors' use of specific and relevant keywords to describe Diversity Science research educates editors, reviewers, and the field. Journals can change their messaging by increasing the pool of inclusive keywords (e.g., see Cultural Diversity and Ethnic Minority Psychology Journal), keep these keywords current with the evolving language in Diversity Science (e.g., BIPOC), include an "other" field to allow for keywords that are not currently offered and that uplift (e.g., systems centered language), and track publication keywords.

8. Define race contextually and conceptually. Race is a political and social construct, independent of biology, that reflects systematic racist practices and inequities (Helms, 2020; Smedley \& Smedley, 2005). Accordingly, authors need to acknowledge the sociopolitical nature of race in text or as a footnote so that readers are clear on the positionality of the author(s) in their examination of this construct. For example, in manuscripts (name withheld) explicitly states: "Race is a political and social construct that often serves as a proxy for the impact of racist practices and structural inequality, it is not a biological variable and thus is examined in the current paper with this premise in mind," which also aligns with

\footnotetext{
2 Examples include racial justice, racism, intersectionality theory, oppression, minority stress, sexism, structural inequality, cultural humility, implicit bias, discrimination, ethnic identity, racial identity, health equity, diversity, and inclusion.
} 
recent changes made to the American Medical Association Manual of Style (AMA, 2020; Flanagin et al., 2021).

9. Require reporting of ethnicity for all participants. Race and ethnicity are often and inappropriately used interchangeably. Ethnicity refers to shared cultural heritage (e.g., ancestry, sense of history, language, religion, foods, and clothing) that distinguishes one group of people from another and is dynamic, varying as a function of context, place, and time (see Ford \& Harawa, 2010). Ignoring participant ethnicity or reporting ethnicity for only one ethnic group (e.g., Hispanic/Latinx/e/a/o) maintains and upholds white supremacist practices in science. Race and ethnicity (e.g., African American, Hmong, Basques, Ashkenazi, Native Hawaiian) should be assessed for all participants and, rather than using binaries such as Hispanic/non-Hispanic, reflect the richness of ethnicities within groups (e.g., Cuban, Mexican). Nationality is another important marker of group heterogeneity that can represent ethnic identity and/or a relationship to a nation state (e.g., Nigerian, Dominican, Korean, French) that researchers should consider, assess and report, particularly when participants may come from different countries. Authors should describe how these designations were determined (e.g., selfidentified, census-defined, identified by parents) to help readers understand potential biases in how these data are reported.

Despite calls to, at a minimum, report and consider finer differentiation within BIPOC groups (Buchanan \& Wiklund, 2020; Hall et al., 2016), 73\% of psychology papers across 11 journals did not provide any data on the race or ethnicity of their participants (DeJesus, et al., 2019). Studies that do so rarely recognize important cultural differences (e.g., not all Black people are African American; Indigenous people include members of specific nations, and may or may not live on reservations). The APA Publication Manual (APA, 2020) and Journal Article Reporting Standards (JARS; Applebaum et al., 2018; Levitt et al., 2018) require the reporting of ethnicity in the Methods section. However, consistent with suggestions in the AMA Manual of Style (Flanagin et al., 2021), more can be required, including careful selection and sensitivity to the labels used, capturing the multiple identities people identify with, reporting who provided the information about participant race and ethnicity, describing the manner in 
which the data was collected (e.g., interview, survey, electronic record, etc.), and discussing the generalizability of study findings to various populations across race, ethnicity, and nationality.

10. Report heterogeneity within BIPOC populations. Intersections among race, ethnicity, gender, social class, sexual orientation, and other identities shape lived experiences and generality of study findings, as such, reporting detailed participant demographics is essential for meeting baseline standards of good science (Buchanan \& Wiklund, 2021). It is critical that demographic information be reported such that intersecting identities are easily identified, moving beyond tallies across gender and separately across race to identify the number of individuals within each category (e.g., Black women, Asian men, and expanded categories including social class, sexual orientation, and gender identity) and examine if findings are similar across these groups (Flanagin et al., 2021). Group heterogeneity should be reported, considered analytically as appropriate (Flanagin et al., 2021; see Suyemoto et al., 2016 for an example of a detailed demographic questionnaire), and justified when collapsed for analyses. AMA publication standards require justification for how and why demographic information was collected and that all available demographic details be provided in the paper or online supplement (Flanagin et al., 2021).

\section{How Science is Reviewed}

Racism and white supremacy can be endemic throughout scientific reviews of psychology research. All players within the review process (i.e., authors, reviewers, editors, and publishers) need to deepen their understanding of the many ways that white supremacist assumptions are maintained and systemic racism is proliferated. Biases in the peer-review process are prevalent, yet ignored, with significant impacts on publication (Lee et al., 2013; King et al., 2018; Stanley, 2007). Despite masked review, these biases disproportionately affect BIPOC scholars and research relevant to BIPOC communities in publication and grant reviews.

Addressing these patterns requires confronting existing power dynamics in psychological science that contribute to the (in)visibility of some scholars and scholarship over others (Buchanan \& Settles, 2019; Settles, Warner et al., 2020). Editors have power to determine what will be published, senior scholars have power over junior scholars (i.e., given a greater likelihood of serving as reviewers and having their comments more heavily weighted), and professional society leaders have power to select 
journal editors and define journal foci. In each case, white investigators likely hold power over BIPOC scholars given that the scientific review process was created by whites and implicitly sustains white privilege. Without acknowledging the power disparities undergirding scientific research and publishing, proposed solutions will be ineffective in ending white supremacy in psychological science. As such, several changes are proposed to dismantle racism and white supremacy in the scientific review process.

\section{Establish systems to examine inequities in reviews and their impact on BIPOC research. As the field} adopts a new culture, language, and awareness of its complicity in maintaining white supremacy, all parties can vigilantly monitor processes that can create inequalities in psychological science. Editors and publishers have considerable power in establishing and enforcing standards in the field and need to be mindful that progress is independent of individual priorities which can change with editorial leadership turnover. To create systemic change, processes and systems can be put in place that supersede the individuals involved and actively monitor ways that the publication process facilitates bias in research. This could be done scientifically, using objective measurements, quantitative data, peer review, and accountability within associations or publishers that publish journals. Such processes have the added benefit of potentially highlighting unknown problems. For example, discovering differences in the outcomes of papers with BIPOC foci may reveal systematic differences in how those papers are reviewed, and invite novel solutions (see suggestions below). Similarly, establishing systems to recognize and protocols for responding to biased reviewer comments, allows editors to give feedback to reviewers and an opportunity to revise reviews before sending them to authors (Sue \& Torino, 2005). This reduces the perpetuation of bias that BIPOC scholars may experience during the review process and the editors' complicity in such bias by presenting these comments as part of their review process.

12. Implement changes at the top that address ongoing racism in the review process. Ongoing patterns of systemic racism can affect the trajectory of entire scientific disciplines. It is recommended that publishing outlets, and the societies/professional associations that sponsor journals, create and maintain a process (e.g., ombudsperson, anonymous reporting avenue) that allows scholars to report practices that maintain epistemic oppression. Moreover, professional societies can examine existing journal and divisional leadership and move toward developing new procedures that promote equitable 
practices. Specifically, professional societies and review committees should evaluate editor candidates based on their track records of dismantling white supremacy, evaluate candidates' ability to move the journal toward a more equitable review process, and require a diversity statement in their application package - one that explicitly outlines how they will increase BIPOC presence in authorship, editorial membership, and content and how they have handled issues of racism in the past. Publishers can evaluate journals and provide incentives (e.g., give editors more page space or monetary incentives) through metrics (such as those in the diversity accountability index (Buchanan et al., 2021) at https://psyarxiv.com/zp9em), and develop policies and sanctions for ongoing racism in the review process.

13. Create a system for authors to rate reviews and reviewers. Racism in the editorial review process is rampant; yet there are no formal mechanisms for authors to give feedback when reviews reflect biased or racist assumptions. For example, one co-author (name withheld) regularly receives comments such as "One of the weaknesses of the study is the inclusion of only English-speaking Hispanics/Latinx which significantly limits the generalizability to a small proportion of Hispanics/Latinx in the U.S." This statement relies on the problematic stereotype that the majority of Hispanics/Latinx in the U.S. do not speak English. ${ }^{3} \mathrm{~A}$ formal feedback system would allow authors to bring such biased statements to the attention of the editorial team. Similarly, it is important that authors have an opportunity to evaluate how well reviewers and editors understand issues related to systemic racism and diversity science and the helpfulness, timeliness, and usefulness of reviews. This would provide a mechanism for all authors (and allow BIPOC authors, in particular) to evaluate how well reviewers understand a manuscript's content and inform editors of the (in)appropriateness of the reviews received. Understanding how the review process can be improved and where it works well can help editors and publishers serve the needs of readers and authors, provide data on reviewers, and document the frequency of racism within reviews, while offering a way for journals to set benchmarks for improving the review process.

\footnotetext{
${ }^{3}$ In 2013, 89\% of U.S. born Hispanics/Latinx spoke English proficiently and 34\% of Hispanics/Latinx born outside the U.S. speak English proficiently due to English language requirements in educational systems of Spanish-speaking countries (Krogstad et al., 2015).
} 
This recommendation has several additional benefits. First, de-identified, aggregated feedback can provide helpful feedback to reviewers. Second, this information will assist editors in selecting reviewers for studies examining BIPOC samples. Third, it can empower authors to be more involved in the editorial process and increase the likelihood that authors get useful feedback. Finally, such efforts could enhance retention among scientists who work with BIPOC participants and communities.

14. Address discriminatory and biased feedback within the review process. Authors, reviewers, or editors sometimes include discriminatory and microaggressive comments that invalidate scientists' professional expertise, theoretical innovation, lived experience, and/or importance of their contributions. The consequences of such behavior are significant for any scholar, but may be especially damaging when they target a BIPOC student or early career scholar who then justifiably feels their work, and potentially they themselves, are unwelcome, misunderstood, and rejected by their peers.

There are numerous examples that illustrate these processes; one is provided below to illustrate behaviors that reflect white supremacy in psychological science and contribute to the disproportionately low representation of BIPOC scientists in psychology. This example involved a BIPOC student first-author whose manuscript examined stressful experiences within an Indigenous sample. The introduction of the paper discussed historical traumas (i.e., forced removal from homelands, legal prohibitions to engage in spiritual practices) that are relevant to systemic disparities within the indigenous community today. A reviewer remarked that these historic traumas are "irrelevant," "unrelated to the study" and reflected "very old issue(s)" - dismissing the potential that these issues continue to affect the conditions under which many Native American communities endure today, communicating indifference towards systemic racism and dismissing the continued mistreatment and genocide of indigenous peoples. The action editor did not address this reviewer's comments, nor provided guidance on how the authors should address the comments. Notably, the student author was personally offended by the reviewer's comments, questioned the field's openness to research on Native American populations, and questioned whether knowledgeable reviewers with sufficient expertise with BIPOC populations would ever review her work. Thus, it is recommended that editors evaluate reviews. Further, action editors should also receive training on best practices for responding to reviewers when discriminatory 
comments are made that reinforce white supremacy. Preventing harmful reviewer feedback from deterring research on BIPOC populations is a necessary strategy to recruit and retain BIPOC scientists in psychological science.

15. Increase the number of BIPOC journal reviewers and editors. Concepts such as 'no taxation without representation' and 'being judged by a jury of one's peers' are based on a foundational belief that those subject to laws and policies--and standards of a field--should see themselves reflected in those creating the laws, policies, and standards. This is no less true in psychological science and in the peer review process. BIPOC scholars occupy social positions that provide them with life experiences, perspectives, and values that influence their epistemic values, research topics and populations of interest (Bernal \& Villalpando, 2002; Gonzales, 2018). Appropriate evaluation of their work requires that evaluators are representative of BIPOC groups so they may be judged by peers with similar understanding of the context in which the scholarship originated.

Representation in the review and editorial process impacts the outcome of submitted manuscripts. Roberts and colleagues (2020) queried over 26,000 empirical psychology publications published in top-tier psychology journals between 1974-2018 and found that only 5\% focused on race (ranging from less than $1 \%$ in cognitive psychology and a high of $8 \%$ in developmental psychology). When the editors of these journals were white, the proportion of such articles dropped precipitously to less than $4 \%$, but under BIPOC editors, the number of published studies examining race nearly tripled (11\%). To create equity in publication outcomes, peer reviewers and editors at all levels must include members from BIPOC communities in sufficient numbers for them to have influence in decision-making. "Desk-rejection" (i.e., preemptively rejecting a manuscript for poor fit to the journal aims, or for failure to meet specific journal standards, without soliciting peer review) of BIPOC papers because of insufficient reviewer expertise is unacceptable, and maintains systemic racism. Journals could hold themselves accountable by collecting information on the diversity of the editorial board, working to increase the diversity of their editorial board, and publishing pictures (in addition to names) of their editorial board members on their website (Kelly \& Patrice, 2019; Neblett, 2019). Journals can display 
pictures of flags from the countries where editorial board members come from as a way to showcase international diversity.

16. Include BIPOC community experts on editorial advisory boards. Community-based partnerships can improve research quality, practice, implementation in real-world settings, and effectiveness in creating equitable changes in power structures (Michener et al., 2012). Partnerships with community members can strengthen research protocols, ask questions with greater relevance to impacted communities and illuminate common research oversights (Hardy et al., 2016; Hiratsuka et al., 2020). Given their benefit to research processes, community members from BIPOC communities should also be involved in the evaluation of research for publication given their expertise and lived experiences directly related to research findings. Publishers can extend beyond traditional academic networks to fill editorial advisory boards and ensure BIPOC community members are included on advisory boards. This will allow community members to provide feedback to editors, reviewers, and researchers about bias and how it impacts their community and to have a voice regarding needed and relevant research for these communities. There may be creative ways for community members to participate in the review process if not in a critique on theories or methods, then perhaps for comments that may guide the discussion and conclusions reached from research studies. Note that those with lived experience similarly have been asked to comment and consult on research studies in other professional activities (i.e., within conference symposia led by scientists) and opportunities to consider this input in the review process as advisory to action editors may strengthen BIPOC studies as well as psychological science more broadly. 17. Provide diversity, equity, and inclusion-related training to the entire editorial team. Unlearning biases is an ongoing process that requires intentional action. Training for all involved within the peer review process is necessary and may facilitate that all members have similar knowledge and expectations regarding diversity, equity and inclusion. All editors, associate editors, and reviewers should participate in ongoing training on topics such as, explicit/implicit bias, bias in the peer review system, soliciting and valuing BIPOC reviewers' comments, understanding current needs and gaps in diversity science literature, confronting one's own biases, and reconciling evidence that may conflict 
with one's feelings, with scaffolded training over time to build upon editors' and editorial board members' development.

18. Mentor BIPOC scholars to engage in editing/reviewing as a vital professional service. High impact journals typically rely on relatively few BIPOC reviewers (Hartmann et al., 2013), contributing to disparate outcomes for publications (i.e., epistemic exclusion; Dotson, 2012; Settles, Jones et al, 2020). Educators can significantly affect this issue by mentoring scholars to see journal reviewing as an essential service not only to promote research careers, but to reduce white supremacy in psychological science. This may occur through the use of mentored (or team-based) reviews at the individual lab level, through journal-based mentored review programs (e.g., see Journal of Pediatric Psychology), or by establishing formal mentorship programs within professional societies to cultivate the development of future BIPOC associate editors and editors. Such programs may be especially useful for professional development regarding the editorial process while also providing support for the myriad of biases faced by BIPOC scholars (e.g., see the National Science Foundation ADVANCE Scholars Programs; Carter-Sowell et al., 2019). Thus, when journals invite BIPOC scholars to serve as guest editors for a special issue (or to co-edit with a senior scholar) this can serve as a vital training opportunity and reviewers can be encouraged to include BIPOC trainees in the review process.

19. Recognize the contributions of BIPOC editorial board members. Serving on editorial boards and reviewing manuscripts for a journal is a vital service for the advancement of science. Given that editors are overwhelmingly white (Greco et al., 2016; Taylor et al., 2020), positive reinforcement can be an important tool for engaging and retaining new BIPOC scholars in the editorial process. Various agencies such as the National Academy of Sciences, Engineering, and Medicine and the Ford Foundation, write personalized letters for scholars identifying service contributions and impact on the field and send the letters to those evaluating the scholar (e.g., department chair, dean, promotion and tenure committee). These letters serve to recognize and reward individuals for their national service contributions, which may be especially vital to the careers of BIPOC editorial board members and reviewers. A disproportionate majority of BIPOC psychologists pursue clinically-oriented jobs (Becker, 2019). Thus, journals may need to employ additional effort and incentives to engage the few BIPOC scholars available 
to provide reviews (e.g., free access to a society's webinars or professional development resources), particularly given the generally higher service burden for BIPOC scholars, and particularly high burden in review requests (e.g., increased reviewer load, reviewing a wider range of topic areas that touch on diversity) that are not compensated.

20. Invite BIPOC scholars to present counter commentary. When manuscripts with the potential to reinforce white supremacy or to disparage BIPOC individuals and communities (e.g., focusing on lowincome communities) are considered for publication, editors can remain complicit, or invite BIPOC scholars to publish a commentary. Commentaries offer opportunities to educate the field on new ways to reconsider data, provide a forum to introduce alternate theories or perspectives, challenge aspects of the article, extend the position the author has taken, connect the research to the experiences of diverse cultures, and elevate the voices of BIPOC scholars (see Buchanan \& Wiklund, 2021). Further, commentaries can serve to recognize BIPOC scholars as experts, and promote scholarship on communities of color by people of color. Sadly, there are numerous examples of manuscripts published by white authors that disparage BIPOC communities or diversity science, with no opportunity for BIPOC scholars to comment on the science (Fairchild, 2000). Consistent with the previous section, journals should consider ways to compensate scholars for this work, such as granting them free access to the journal for a period of time and providing email recognition of the importance of their work which can be used for promotion, tenure, and merit reviews.

21. Track rejection rates for manuscripts with predominantly BIPOC samples. Journal editors have an opportunity to elevate, or perhaps inadvertently to systematically exclude, papers from publication. Given archival and experimental findings that diversity-related research is more likely to be rejected than accepted (King et al., 2018), editors have reason for concern that their journal's review processes also may perpetuate systemic biases against such research. This can negatively impact the promotion process for BIPOC scholars and perpetuate biases against BIPOC scholars. It is recommended that journal editors track the topics and population of papers that are rejected, either with or without peer review, and report these data annually for accountability. This will provide researchers with data on 
which outlets may be receptive to psychological science from BIPOC perspectives and helps publishers examine the systematic exclusion of BIPOC scholarship.

22. Empower authors to educate the field about race. Most scientists learn to "market" their research to increase its applicability, impact, and to emphasize its need. For psychological science conducted by BIPOC scholars, or related to psychological processes relevant within BIPOC populations, these marketing skills are more complex for several reasons. First, investigators need to not only highlight research as understudied and important, but to also skillfully critique why prior research conducted by white authors or on white populations may not be applicable. This is a challenging task given inherent differences in power between white and BIPOC investigators within the field. Second, the marginalization of prior work on BIPOC populations (i.e., within "specialty" journals) is read less frequently by white investigators, and the paucity of BIPOC scholars in the field, leaves few qualified experts available to evaluate research on BIPOC populations. Therefore, scholars must provide substantial didactic material to explain the significance of research being conducted. Last, it is important to recognize that these added burdens require manuscript preparation skills that may not be discussed or taught in most doctoral programs. Consequently, it is recommended that training programs for psychological scientists explicitly discuss and train students to explain how studies on BIPOC populations contribute to the literature, to educate readers and reviewers about important biases in prior work, and to skillfully rebut comments in the editorial process that may maintain white supremacy or unfairly penalize research by BIPOC investigators and on BIPOC populations.

\section{How Science is Disseminated}

Research dissemination priorities can reflect the pervasiveness of racism and white supremacy in psychological science. Medicine and psychological science have a lengthy history of exploiting BIPOC communities and can counter this history by, at a minimum, disseminating research in ways that reach beyond a journal's readership to benefit public audiences and BIPOC communities specifically. High quality public dissemination requires skill sets, writing, and methods that differ from academic writing. Below are dissemination practices to upend racism and white supremacy in psychological science. 
23. Report and reward community-based dissemination. When scholars submit their work to academic journals they can be penalized for public dissemination efforts that are archived online. At best, originality algorithms suggest a diminished novelty of work previously disseminated in community presentations and non-academic publications and at worst, imply scholars plagiarized their work. These practices and the fear that a manuscript may be negatively evaluated because of community dissemination may stymie public dissemination efforts and contribute to systemic biases that reduce the chances that research with BIPOC populations, particularly when community based, will be accepted and recognized as novel contributions to the field. To reverse this trend, journals should celebrate broader dissemination efforts by welcoming research disseminated for public and community audiences as novel to academic publications and adjusting originality indices so community dissemination efforts are not penalized.

\section{Create public-focused dissemination strategies that extend findings to BIPOC communities.}

Scientific contributions are increasingly measured by direct dissemination to communities and the public (e.g., using Altmetrics) in addition to citations within the scientific literature. Journals can embrace and build upon this trend. Dissemination to BIPOC communities can and should come in many formats, such as practice-oriented journals that provide easily digestible research findings and have clear implications for communities that psychologists serve, and/or open-access journals which are more readily accessible to community partners and news media outlets. Further, social media and newsletters that actively disseminate research to communities that can benefit from them should be championed. Research briefings that are tailored for policy makers and health officials should be encouraged. Infographics that are inclusive and relevant to BIPOC communities, podcasts that reach diverse audiences, and emerging formats that extend the reach of science should be encouraged by journals. Journals can require that authors submit a separate abstract written for public audiences that summarizes findings in easily understood language and without jargon. Publishers should ensure they have dedicated staff with specialized skills to broadly disseminate scientific findings across a variety of platforms that are broadly accessible, easily understood, and relevant to BIPOC communities. 
25. Collaborate with community partners for dissemination. One of the principles of CBPR involves collaborating with community partners at all stages of the research process (Israel et al, 2010), including dissemination of research findings. Scientists are notoriously poor at sharing science with the public. Thus, community partners should be an integral part of decision-making for dissemination. When BIPOC community partners are involved in all stages of the research process, they can readily share knowledge, resources, and expertise about how, where, and to whom findings should be disseminated in order to have meaningful impact. Working together as co-authors to produce articles in scientific and nonscientific outlets facilitates publications that are written and presented in ways that move research into the hands and minds of the public. When community partners are co-authors, dissemination to the communities impacted by research can occur more seamlessly and naturally.

\section{Accountability}

Many scientists, in psychology and other disciplines, have begun discussing processes that have unfairly benefitted white scholars over BIPOC scientists, advanced research on white populations over populations of color, and maintained a default reliance on scientific theories and methods originating from white-dominated perspectives as compared to perspectives that represent and celebrate diverse communities. The discipline of psychological science has an opportunity to lead within the broader intellectual community by not only identifying and speaking about anti-racism, but also developing new scientific practices in meaningful ways, with accountability. Too often, BIPOC communities have heard promises of reform, and well-meaning plans towards inclusion, only to have hopes unfulfilled as whiteness resumes a default norm. This unprecedented moment in history will lead to meaningful change only if all stakeholders in the science community are held accountable to recent verbal commitments to change.

Several steps are recommended to ensure accountability. First is the creation of a reliable and valid instrument that can be used to measure anti-racist practices, such as those that have been discussed in this paper. To assist in this task, an example of an accountability instrument, organized by the sections and 25 suggestions offered above is available at https://psyarxiv.com/zp9em (Buchanan et al., 2021). Posting this draft in an open science format, before peer review, offers two opportunities. 
First, it provides a basis for immediate comment and debate within the field while long-overdue attention is being dedicated to diversity, equity, and inclusion (DEI) initiatives. Note that while scientific processes led by white scholars may traditionally involve editors and publishers (who currently are predominantly white) to weigh in on a measure designed to promote accountability, DEl efforts are better reflected by a field-wide dialogue inviting all voices to contribute, even at this initial stage. Also, posting this instrument (or revisions thereof) on an open science platform allows pilot testing immediately by journal editors and publishers, to determine what adaptations may be needed for maximal generality within the field.

A second step towards ensuring accountability will be to determine a baseline of current practices to establish benchmarks reflecting current practices. It is recommended that these data are made available publicly to identify practices that might be addressed within specific journals, or perhaps by broader systemic changes. Assessments can be repeated to measure progress over time, with ongoing public reports serving as an external accountability to ensure that journals (scientific societies, associations, publishers) continue to address diversity issues. High scores on standardized accountability instruments could be championed, similar to journal impact factors, as proud accomplishments that earn a journal greater respect, and increases submissions and academic prestige.

Data used to evaluate anti-racist practices within journals likely will reveal specific areas that are at different points within the continuum of change, or different journal practices that have led to varying levels of success. These data thus will provide a basis for critical, evidence-based dialogue that can promote ongoing change, and an iterative and empirically-based process to guide progress over the next several decades. Last, it would be useful for scientific societies, associations, and publishers to discuss potential remedial or disciplinary action to resolve poor practices (i.e., continued poor accountability scores) where scientists have been treated inequitably, or when science has poorly reflected diversity.

\section{Conclusion}

Today there is an opportunity to greatly improve psychological science by dismantling white supremacy and epistemic oppression in the discipline. This paper discussed action and accountability steps for one part of a much larger set of issues that must be addressed in the field of psychology. This paper 
specifically addresses four processes in psychological science and 25 concrete, actionable steps, with a focus on the next, but not the only, steps that are necessary to eliminate white supremacy. It is noted that although this paper aimed to illustrate the systemic nature of change to achieve equity, inclusion, and diversity by highlighting simultaneous actions necessary across a variety of stakeholders, this discussion is by no means exhaustive; granting agencies, institutes of higher education are among many other systems that require change to fully eliminate white supremacy in the field's scientific endeavors. In addition, intersectionality and attention to other systems that impact inequality must also be addressed. Some of the issues discussed above apply to supremacy afforded to scholars who are male, cis-gender, heterosexual, able-bodied, and to populations that do not include consideration of sexual, gender, neuro-diversity, or variation in ability. Each of these blind spots within psychological science, in addition to white supremacy, require dismantling and have plagued the discipline for far too long. Psychological science aims to understand, and guide efforts towards improving the thoughts, attitudes, behavior, emotions, and abilities of all humans, and only when epistemic oppression is eliminated will the discipline have an opportunity to achieve this goal.

\section{References}

Alexander-Floyd, N. (2012). Disappearing Acts: Reclaiming Intersectionality in the Social Sciences in a Post-Black Feminist Era. Feminist Formations, 24(1), 1-25. http://www.jstor.org/stable/23275087 American Psychological Association. (2018). Demographics of the U.S. psychology workforce: Findings from the 2007-16 American Community Survey. Washington, DC: Author.

https://www.apa.org/workforce/publications/16-demographics/report.pdf

American Psychological Association. (2020). Publication manual of the American Psychological Association (7th ed.). Washington, DC: American Psychological Association. https://doi.org/10.1037/0000165-000

Appelbaum, M., Cooper, H., Kline, R. B., Mayo-Wilson, E., Nezu, A. M., \& Rao, S. M. (2018). Journal Article Reporting Standards for Quantitative Research in Psychology: The APA Publications and Communications Board Task Force report. American Psychologist, 73, 3-25. https://doi.org/10.1037/amp0000191 
Attridge, J., Zimmerman, D., Davis, J., \& Rolin, S. (2020). A-238 Psychometric equivalence of prorated Boston Naming Test scores after noose item removal. Archives of Clinical Neuropsychology, 35(6), 1022. https://doi.org/10.1093/arclin/acaa068.238

Baker, T. A., Buchanan, N. T., Corson, N. (2008). Factors influencing chronic pain intensity in older Black women: Examining depression, locus of control and physical health. Journal of Women's Health, 17, 869-878. http://dx.doi.org/10.1089/jwh.2007.0452

Becker, C. B. (2019). Can we do better? Addressing structural barriers to increasing diversity in clinical psychology. Clinical Science Newsletter, 23(3), 2-4.

Bernal, D. D. \& Villalpando, O. (2002). An apartheid of knowledge in academia: The struggle over the "legitimate" knowledge of faculty of color. Equity \& Excellence in Education, 35(2), 169-180. https://doi-org.proxy.lib.umich.edu/10.1080/713845282

Bertolero, M. A., Dworkin, J. D., David, S. U., Lloreda, C. L., Srivastava, P., Stiso, J., ... \& Bassett, D. S. (2020). Racial and ethnic imbalance in neuroscience reference lists and intersections with gender. BioRxiv. https://www.biorxiv.org/content/10.1101/2020.10.12.336230v1

Buchanan, N. T. \& Ormerod, A. J. (2002). Racialized sexual harassment in the lives of African American Women. Women \& Therapy, 25, 107-124. http://dx.doi.org/10.1300/J015v25n03 08

Buchanan, N. T., Perez, M., Prinstein, M. J., \& Thurston, I. B. (2021). Diversity Accountability Index for Journals (DAI-J): Increasing awareness and establishing accountability across psychology journals [unpublished manuscript]. PsyArXiv. https://psyarxiv.com/zp9em

Buchanan, N. T. \& Settles, I. H. (2019). Managing (in)visibility and hypervisibility at work. Journal of Vocational Behavior, 113, 1-5. https://doi.org/10.1016/j.jvb.2018.11.001

Buchanan, N. T., Settles, I. H., Wu, I. H. C., \& Hayashino, D. S. (2018). Sexual harassment, racial harassment and well-being among Asian American women: An intersectional approach. Women \& Therapy, 41, 261-280. https://doi.org/10.1080/02703149.2018.1425030

Buchanan, N. T. \& Wiklund, L. (2020). Why clinical science must change or die: Integrating intersectionality and social justice. Women \& Therapy, 34(3-4), 309-329. https://doi.org/10.1080/02703149.2020.1729470 
Buchanan, N. T. \& Wiklund, L. (2021). Intersectionality Research in Psychological Science: Resisting the Tendency to Disconnect, Dilute, and Depoliticize. Research on Child and Adolescent Psychopathology, 49, 25-31. https://doi.org/10.1007/s10802-020-00748-y

Burlew, A. K., Peteet, B. J., McCuistian, C., \& Miller-Roenigk, B. D. (2019). Best practices for researching diverse groups. American Journal of Orthopsychiatry, 89(3), 354-368. https://doi.org/10.1037/ort0000350

Card, D., \& DellaVigna, S. (2012) How Do Authors Respond to Page Limits? https://eml.berkeley.edu/ sdellavi/wp/pagelimits12-10-08.pdf

Carter-Sowell, A. R., Vaid, J., Stanley, C. A., Petitt, B., \& Battle, J. S. (2019). ADVANCE Scholar Program: Enhancing minoritized scholars' professional visibility. Equality, Diversity, and Inclusion: An International Journal, 38(3), 305-327. https://doi.org/10.1108/EDI-03-2018-0059

Centola, D., Becker, J., Brackbill, D., \& Baronchelli, A. (2018). Experimental evidence for tipping points in social convention. Science, 360(6393), 1116-1119. https://doi.org/10.1126/science.aas8827

Chávez, V., Duran, B., Baker, Q. E., ... \& Wallerstein, N. (2008). The dance of race and privilege in CBPR. In M. Minkler \& N. Wallerstein (Eds.) Community Based Participatory Research for Health. $2^{\text {nd }}$ Ed. Jossey-Bass.

Chakravartty, P., Kuo, R., Grubbs, V., \& Mcllwain, C. (2018) \#CommunicationSoWhite. Journal of Communication, Volume 68, (2),254-266, https://doi.org/10.1093/joc/jqy003

Chen, C. H. (2011). The major components of corporate social responsibility. Journal of global responsibility, 2(1), 85-99. https://doi.org/10.1108/20412561111128546

Cundiff, J. L. (2012). Is mainstream psychological research "womanless" and "raceless"? An updated analysis. Sex Roles, 67(3), 158-173.https://doi-org.proxy2.cl.msu.edu/10.1007/s11199-012-0141-7

Dawson, J., Coggins, N. L., Dolechek, M., \& Fosado, G. (2020). Toolkits for Equity: An Antiracist Framework for Scholarly Publishing. Serials Review, 46(3), 170-174. https://doi.org/10.1080/00987913.2020.1806653 
DeJesus, J. M., Callanan, M. A., Solis, G., \& Gelman, S. A. (2019). Generic language in scientific communication. Proceedings of the National Academy of Sciences, 116(37), 18370-18377. https://www.pnas.org/content/pnas/116/37/18370.full.pdf

Diaz, I., \& Bergman, M. E. (2013). It's not us, it's you: Why isn't research on minority workers appearing in our "top-tier" journals?. Industrial and Organizational Psychology, 6(1), 70-75.

Dotson, K. (2012). A cautionary tale: On limiting epistemic oppression. Frontiers: A Journal of Women Studies, 33(1), 24-47. https://www.jstor.org/stable/10.5250/fronjwomestud.33.1.0024

Dzirasa, K. (2020). Revising the a Priori Hypothesis: Systemic Racism Has Penetrated Scientific Funding. Cell, 183(3), 576-579. https://doi.org/10.1016/i.cell.2020.09.026

Erosheva, E. A., Grant, S., Chen, M. C., Lindner, M. D., Nakamura, R. K., \& Lee, C. J. (2020). NIH peer review: Criterion scores completely account for racial disparities in overall impact scores. Science Advances, 6(23), eaaz4868. https://doi.org/10.1126/sciadv.aaz4868

Fairchild, H. H. (2000). African American psychology. In A. E. Kazdin (Ed.), Encyclopedia of psychology (pp. 92-99). American Psychological Association.

Flanagin, A., Frey, T., Christiansen, S. L., \& Bauchner, H. (2021). The Reporting of Race and Ethnicity in Medical and Science Journals: Comments Invited. JAMA. https://doi.org/10.1001/jama.2021.2104

Ford, C. L., \& Harawa, N. T. (2010). A new conceptualization of ethnicity for social epidemiologic and health equity research. Social Science \& Medicine, 71(2), 251-258. https://doi.org/10.1016/j.socscimed.2010.04.008

Garcia, D. C. F., Gattaz, C. C., \& Gattaz, N. C. (2019) The Relevance of Title, Abstract and Keywords for Scientific Paper Writing. Revista de Administracão Contemporânea, 23(3). https://doi.org/10.1590/1982-7849rac2019190178

Ginther, D. K., Schaffer, W. T., Schnell, J., Masimore, B., Liu, F., Haak, L. L., \& Kington, R. (2011). Race, ethnicity, and NIH research awards. Science, 333(6045), 1015-1019. https://doi.org/10.1126/science.1196783 
Gonzales, L. D. (2018). Subverting and minding boundaries: The intellectual work of women. The Journal of Higher Education, 89(5), 677-701. https://doiorg.proxy.lib.umich.edu/10.1080/00221546.2018.1434278

Greco, A. N., Wharton, R. M., \& Brand, A. (2016). Demographics of scholarly publishing and communication professionals. Learned Publishing, 29(2), 97-101. https://doi.org/10.1002/leap.1017

Greenwald, A. G., \& Schuh, E. S. (1994). An ethnic bias in scientific citations. European Journal of Social Psychology, 24, 623-639. https://doi.org/10.1002/ejsp.2420240602

Guthrie, R. V. (2004). Even the rat was white: A historical view of psychology. Pearson Education.

Hall, G. C. N., Ibaraki, A. Y., Huang, E. R., Marti, C. N., \& Stice, E. (2016). A meta-analysis of cultural adaptations of psychological interventions. Behavior Therapy, 47(6), 993-1014.

Hall, G. C. N., \& Maramba, G. G. (2001). In search of cultural diversity: Recent literature in cross-cultural and ethnic minority psychology. Cultural Diversity \& Ethnic Minority Psychology, 7, 12-26. https://doi.org/10.1037/1099-9809.7.1.12

Hardy, L. J., Hughes, A., Hulen, E., Figueroa, A., Evans, C., \& Begay, R. C. (2016). Hiring the experts: best practices for community-engaged research. Qualitative Research, 16(5), 592-600. https://doi.org/10.1177/1468794115579474

Hartmann, W. E., Kim, E. S., Kim, J. H., Nguyen, T. U., Wendt, D. C., Nagata, D. K., \& Gone, J. P. (2013). In search of cultural diversity, revisited: Recent publication trends in cross-cultural and ethnic minority psychology. Review of General Psychology, 17(3), 243-254. https://doi.org/10.1037/a0032260

Held, B. S. (2020). Epistemic violence in psychological science: Can knowledge of, from, and for the (othered) people solve the problem? Theory \& Psychology, 30(3), 349-370. https://doiorg.proxy2.cl.msu.edu/10.1177/0959354319883943

Helms, J. E. (2020). A race is a nice thing to have: A guide to being a white person or understanding the white persons in your life. Cognella.

Henrich, J., Heine, S. J., \& Norenzayan, A. (2010). Most people are not WEIRD. Nature, 466(7302), 29-29. https://doi.org/10.1038/466029a 
Hiratsuka, V. Y., Trinidad, S. B., Ludman, E. J., Shaw, J. L., Burke, W., Robinson, R. F., \& Dillard, D. A. (2020). "You Actually View Us as the Experts in Our Own System": Indigenous-Academic Community Partnership. Progress in Community Health Partnerships: Research, Education, and Action, 14(2), 187-195. https://doi.org/10.1353/cpr.2020.0018

Hoppe, T. A., Litovitz, A., Willis, K. A., Meseroll, R. A., Perkins, M. J., Hutchins, B. I., ... \& Santangelo, G. M. (2019). Topic choice contributes to the lower rate of NIH awards to African-American/black scientists. Science advances, 5(10), eaaw7238. https://doi.org/10.1126/sciadv.aaw7238

Howard, M. C., \& Hoffman, M. E. (2018). Variable-centered, person-centered, and person-specific approaches: where theory meets the method. Organizational Research Methods, 21(4), 846-876. https://doi.org/10.1177/1094428117744021

Israel, B. A., Coombe, C. M., Cheezum, R. R., Schulz, A. J., McGranaghan, R. J., Lichtenstein, R., ... \& Burris, A. (2010). Community-based participatory research: a capacity-building approach for policy advocacy aimed at eliminating health disparities. American Journal of Public Health, 100(11), 20942102. https://doi.org/10.2105/AJPH.2009.170506

Kelly, K., \& Patrice, K. (2019). Incorporating black images and references to increase African American student performance in introductory psychology: A pilot study. Journal of Black Psychology, 45, 5262. https://doi.org/10.1177/0095798418825168

King, E. B., Avery, D. R., Hebl, M. R., \& Cortina, J. M. (2018). Systematic subjectivity: How subtle biases infect the scholarship review process. Journal of Management, 44(3), 843-853. https://doi.org/ $10.1177 / 0149206317743553$

Krogstad, J. M., Stepler, R., \& Lopez, M. H. (2015). English proficiency on the rise among Latinos: U.S. born driving language changes. Pew Research Center, Washington D.C., May. https://www.pewresearch.org/hispanic/2015/05/12/english-proficiency-on-the-rise-among-latinos Lozano, G. A., Larivière, V., \& Gingras, Y. (2012). The weakening relationship between the impact factor and papers' citations in the digital age. Journal of the American Society for Information Science and Technology, 63(11), 2140-2145. https://doi.org/10.1002/asi.22731 
Lee, C. J., Sugimoto, C. R., Zhang, G., Cronin, B. (2013). Bias in peer review. Advances in Information Science, 64(1), 2-17. https://doi.org/10.1002/asi.22784

Leong, F. T., Pickren, W. E., \& Vasquez, M. J. (2017). APA efforts in promoting human rights and social justice. American Psychologist, 72(8), 778-790. https://doi.org/10.1037/amp0000220

Levitt, H. M., Bamberg, M., Creswell, J. W., Frost, D. M., Josselson, R., \& Suárez-Orozco, C. (2018). Journal Article Reporting Standards for Qualitative Primary, Qualitative Meta-Analytic, and Mixed Methods Research in Psychology: The APA Publications and Communications Board Task Force report. American Psychologist, 73, 26-46. https://doi.org/10.1037/amp0000151

Markus, H. R. (2008). Pride, prejudice, and ambivalence: Toward a unified theory of race and ethnicity. American Psychologist, 63(8), 651-670. https://doi.org/10.1037/0003-066X.63.8.651

Matsumoto, D., Grissom, R. J., Dinnel, D. L. (2001). Do between-culture differences really mean that people are different? A look at some measures of cultural effect size. Journal of Cross-Cultural Psychology, 32, 478-490. https://doi.org/10.1177/0022022101032004007

Miller, A. L., Stern, C., \& Neville, H. (2019a). Forging diversity-science-informed guidelines for research on race and racism in psychological science. Journal of Social Issues, 75(4), 1240-1261. https://doi.org/10.1111/josi.12356

Miller, A. L., Stern, C., \& Neville, H. A. (2019b). Introduction to the Special Issue on Race and Racism. Journal of Social Issues, 75(4), 992-1001. https://doi.org/10.1111/josi.12359

Michener, L., Cook, J., Ahmed, S. M., Yonas, M. A., Coyne-Beasley, T., \& Aguilar-Gaxiola, S. (2012). Aligning the goals of community-engaged research: Why and how academic health centers can successfully engage with communities to improve health. Academic Medicine, 87(3), 285-291. https://doi.org/10.1097/ACM.0b013e3182441680

Neblett, Jr., E.W. (2019). Diversity (psychological) science training: challenges, tensions, and a call to action. Journal of Social Issues, 74(4), 1216-1239. https://doi.org/10.1111/josi.12357

O'Reilly, M. (2020, June 5). Systems Centered Language: Speaking truth to power during COVID-19 while confronting racism. Medium. https://medium.com/@meagoreillyphd/systems-centered-language$\underline{\text { a3dc7951570e }}$ 
Osbeck, L. (2018). Values in psychological science: Re-imagining epistemic priorities at a new frontier. Cambridge University Press.

Plaut, V. C. (2010a). Diversity science: Why and how difference makes a difference. Psychological Inquiry, 21(2), 77-99. https://doi.org/10.1080/10478401003676501

Plaut, V. C. (2010b). Diversity science: Who needs it? Psychological Inquiry, 21(2), 168-174. https://doi.org/10.1080/1047840X.2010.492753

Proctor, J. (2020). https://www.saytheirnamesmemorials.com/press. Accessed on November 19, 2020

Roberts, S. O., Bareket-Shavit, C., Dollins, F. A., Goldie, P. D., \& Mortenson, E. (2020). Racial inequality in psychological research: Trends of the past and recommendations for the future. Perspectives on Psychological Science, 15(6), 1295-1309. https://doi.org/10.1177/1745691620927709

Roberts, S. O., \& Rizzo, M. T. (2020). The Psychology of American Racism. American Psychologist. Advance online publication. http://dx.doi.org/10.1037/amp0000642

Rosenthal, L. (2016). Incorporating intersectionality into psychology: An opportunity to promote social justice and equity. American Psychologist, 71(6), 474-485. https://doi.org/10.1037/a0040323

Salter, P. S., Adams, G., \& Perez, M. J. (2018). Racism in the structure of everyday worlds: A culturalpsychological perspective. Current Directions in Psychological Science, 27(3), 150-155. https://doi.org/10.1177/0963721417724239

Schneider, K. J. (1998). Toward a science of the heart: Romanticism and the revival of psychology. American Psychologist, 53(3), 277-289. https://doi.org/10.1037/0003-066X.53.3.277

Settles, I. H., Jones, M. K., Buchanan, N. T., \& Dotson, K. (2020). Epistemic exclusion: Scholar(ly) devaluation that marginalizes Faculty of Color. Journal of Diversity in Higher Education. Advance online publication. https://doi.org/10.1037/dhe0000174

Settles, I. H., Warner, L., Buchanan, N. T., \& Jones, M. K. (2020). Understanding Psychology's Resistance to Intersectionality Theory using a Framework of Invisibility and Epistemic Exclusion. Journal of Social Issues, 76, 796-812. http://dx.doi.org/10.1111/josi.12403 
Smedley, A., \& Smedley, B. D. (2005). Race as biology is fiction, racism as a social problem is real: Anthropological and historical perspectives on the social construction of race. American Psychologist, 60(1), 16-26. https://doi.org/10.1037/0003-066X.60.1.16

Stanley, C. A. (2007). When counter narratives meet master narratives in the journal editorial-review process. Educational Researcher, 36(1), 14-24. https://doi.org/10.3102/0013189X06298008

Stevens, K. R., Masters, K. S., Imoukhuede, P. I., Haynes, K. A., Setton, L. A., Cosgriff-Hernandez, E., ... \& Eniola-Adefeso, O. (2021). Fund black scientists. Cell, 184(3), 561-565. https://doi.org/10.1016/i.cell.2021.01.011

Sue, D. W., \& Torino, G. C. (2005). Racial-cultural competence: Awareness, knowledge, and skills. In R. T. Carter (Ed.), Handbook of racial-cultural psychology and counseling: Training and practice. Wiley Inc.

Syed, M., Santos, C., Yoo, H. C., \& Juang, L. P. (2018). Invisibility of racial/ethnic minorities in developmental science: Implications for research and institutional practices. American Psychologist, 73(6), 812-826. https://doi.org/10.1037/amp0000294

Syed, M., \& McLean, K. C. (2021). Master Narrative Methodology: A Primer for Conducting StructuralPsychological Research. Cultural Diversity and Ethnic Minority Psychology. Advance online publication. https://doi.org/10.31234/osf.io/8d7t9

Suyemoto, K. L., Erisman, S. M., Holowka, D.W., Fuchs, C., Barrett-Model, H., ... \& Roemer, L. (2016). UMass Boston comprehensive demographic questionnaire, revised. In Wadsworth, L. P., et al., (2016). Ways to boost your research rigor through increasing your cultural competence. The Behavior Therapist, 39, 83-89.

Taylor, S., Spilka, S., Monahan, K., Mulhern, I., \& Wachter, J. (2020). Evaluating equity in scholarly publishing. Learned Publishing, 33(4), 353-367. https://doi.org/10.1002/leap.1301

Teo, T. (2008). From speculation to epistemological violence in psychology: A critical-hermeneutic reconstruction. Theory \& Psychology, 18, 47-67. https://doi.org/10.1177/0959354307086922

Thalmayer, A. G., Toscanelli, C., \& Arnett, J. J. (2020). The neglected 95\% revisited: Is American psychology becoming less American? American Psychologist, 76(1), 116-129. https://doi.org/10.1037/amp0000622 
Valrie, C., Thurston, I., \& Santos, M. (2020). Addressing Health Disparities in Pediatric Psychology. Journal of Pediatric Psychology, 45(8), 833-838. https://doi.org/10.1093/jpepsy/jsaa066

Wang, Q. (2016). Why should we all be cultural psychologists? Lessons from the study of social cognition. Perspectives on Psychological Science, 11, 583-596. https://doi.org/10.1177/1745691616645552

Winston, A. S. (2020). Why mainstream research will not end scientific racism in psychology. Theory \& Psychology, 30(3), 425-430. https://doi.org/10.1177/0959354320925176

Zuberi, T., \& Bonilla-Silva, E. (2008). White logic, white methods: Racism and methodology. Rowman \& Littlefield Publishers.

Zurn, P., Bassett, D. S., \& Rust, N. C. (2020). The citation diversity statement: a practice of transparency, a way of life. Trends in Cognitive Sciences, 24(9), 669-672. https://doi.org/10.1016/i.tics.2020.06.009 